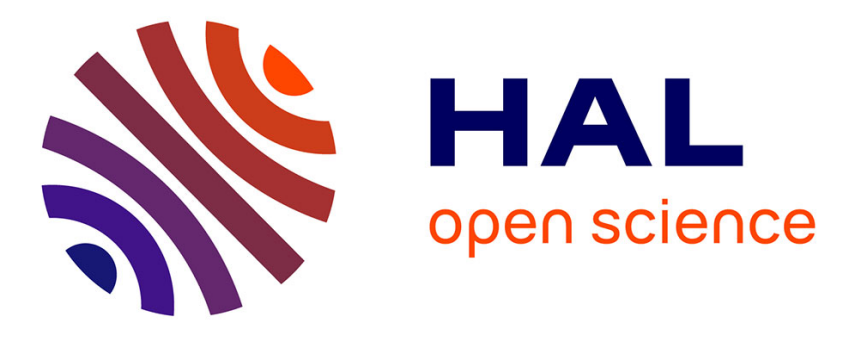

\title{
Development of workflow task analysis during cerebral diagnostic angiographies: Time-based comparison of junior and senior tasks
}

Jean-Christophe Gentric, Brivael Trelhu, Pierre Jannin, Laurent Riffaud, Jean-Christophe Ferré, Jean-Yves Gauvrit

\section{To cite this version:}

Jean-Christophe Gentric, Brivael Trelhu, Pierre Jannin, Laurent Riffaud, Jean-Christophe Ferré, et al.. Development of workflow task analysis during cerebral diagnostic angiographies: Time-based comparison of junior and senior tasks. Journal de Neuroradiologie / Journal of Neuroradiology, 2013, 40 (5), pp.342-347. 10.1016/j.neurad.2013.01.002 . inserm-00845190

\section{HAL Id: inserm-00845190 https://www.hal.inserm.fr/inserm-00845190}

Submitted on 18 Dec 2015

HAL is a multi-disciplinary open access archive for the deposit and dissemination of scientific research documents, whether they are published or not. The documents may come from teaching and research institutions in France or abroad, or from public or private research centers.
L'archive ouverte pluridisciplinaire HAL, est destinée au dépôt et à la diffusion de documents scientifiques de niveau recherche, publiés ou non, émanant des établissements d'enseignement et de recherche français ou étrangers, des laboratoires publics ou privés. 


\section{Original Article}

\section{Development Of Workflow Task Analysis during Cerebral}

Diagnostic Angiographies; Time-Based Comparison of Junior and Senior Tasks.

Développement d'une méthode d'analyse de workflow des artériographies

cérébrales: comparaison de la durée des actions de juniors et de seniors.

Jean-Christophe Gentric a, b, c, d, corresponding author

Brivael Trelhu b, c, d,

Pierre Jannin b, c, d,

Laurent Riffaud b, c, d, e,

Jean-Christophe Ferré b, c, d, f,

Jean-Yves Gauvrit b, c, d, f, corresponding author

a - Department of radiology, Brest university hospital, Brest, France

b - Inserm, U746, faculty of medicine, Rennes, France

c - INRIA, VisAGeS Unit/Project, Rennes, France

d - CNRS, UMR 6074, IRISA, university of Rennes 1, Rennes, France

e - Department of neurosurgery, Rennes university hospital, Rennes, France

f - Department of neuroradiology, Rennes university hospital, Rennes, France

Corresponding authors :

Jean-Christophe Gentric jcgentric@gmail.com

Jean-Yves Gauvrit jean-yves.gauvrit@chu-rennes.fr 


\section{ABSTRACT}

OBJECTIVE: Assessing neuroradiologists' skills in operating room (OR) is difficult and often subjective. A workflow time-based task analysis approach while performing cerebral angiographies has been used.

METHODS: Eight angiographies performed by a senior and 8 performed by a junior were compared. Dedicated software with specific terminology was used to record the tasks. Procedures were subdivided into phases each comprising multiple tasks. Each task was defined as a triplet, associating an action, an instrument, and an anatomical structure. The duration of each task was the metric. The total duration of the procedure, the duration per task, and the number of times a task was repeated, were isolated. Moreover, we focused on the tasks using fluoroscopy or moving the X-ray system.

RESULTS: The total duration of tasks of the entire procedure was longer for juniors than for seniors $(\mathrm{p}=0.012)$. The mean duration per task for the navigation phase was 86 seconds the junior group and 43 seconds for the senior group ( $\mathrm{p}=0.002$ ). The total and mean durations of tasks involving use of fluoroscopy were longer in the junior group $(p=0.002 \& p=0.033$ ). Concerning the tasks involving table and tube, the total and mean durations of these tasks are longer in the junior group ( $\mathrm{p}=0.019 \& \mathrm{p}=0.082)$.

CONCLUSION: This approach allows for reliable skills assessment in the radiology OR and for comparison of junior and senior competencies during cerebral diagnostic angiographies. This new tool could improve the quality and safety of procedures, and facilitate the learning process of neuroradiologists.

KEY WORDS: Educational Measurement, Professional Competence, Surgical Process Models, Surgical Workflow, Systems Analysis 
Abbreviations:

$\mathrm{OR}=$ operating room 


\section{INTRODUCTION}

Objective assessment of interventional or surgical procedures includes analysis of different components such as technical or cognitive skills, knowledge, communication, mental readiness, ability to cope with stress, decision making, and team work [1]. Such assessment is usually based on simulation systems, such as bench models, live animals, cadavers, or virtual simulators. These systems focus on competency outside the OR [2] and cannot reliably assess the operator due to the lack of clinical realism and evaluation directly in the OR. Deconstructing procedure into a list of activities is part of surgical process modeling and analyzing methodology. Splitting the components of the procedure lead to the possible timeassessment of the deconstructed procedure. This approach allows for example, to compare operators' tasks duration and to highlight tasks or phases where difficulties were encountered leading to lengthen the concerned part of the procedure [3-5]. Additionally, such methodology is not conditioned by the subjective impressions of the operator. It mainly relies on live observation by a trained observer recording phase-segmented procedures made up of a sequence of tasks (i.e. the process or the workflow); each task is then extracted and analyzed. For analysis and comparison, metrics, such as the duration of each task and the number of times that task is performed, are used [6]. Comparisons between operators, techniques $[7,8]$ and protocols based on these metrics thus become quantitative and explicit. This approach was recently used in surgery for multimodal imaging interaction [9], procedure phase time analysis [10], and for assessing technical skills [4]. For example, Riffaud et al. compared junior and senior neurosurgeon's competencies in the OR using workflow task analysis during lumbar disc herniation surgery [11]. Interventional radiology has benefited from this approach when applied to uterine fibroid embolization [10] and nerve and facet blocks [12]. Such tools could improve safety and quality of interventional procedures and could be included in training programs. A major factor in achieving efficiency and competency in interventional 
neuroradiology is experience. Younger neuroradiologists tend to have less experience in performing cerebral angiographies as a result of advances in non-invasive imaging.

To demonstrate the validity of this time-based workflow task analysis approach in cerebral diagnostic angiographies, we applied recommendations from previous studies [13-15] and conducted an exemplary study with an expected logical outcome. It consisted in comparing skills of junior and senior neuroradiologists during procedures. To validate our time-based workflow task analysis for assessing operator skills, we chose to analyze junior and senior competencies in performing cerebral angiography with the assumption that juniors would be slower. To the best of our knowledge, this is the first time workflow task analysis has been used to study cerebral diagnostic angiographies.

\section{METHODS}

Procedures and Participants

\section{Patients}

The patients selected for the study had no distinguishing features. Of the 20 patients, 10 were male and 10 female, with a mean age of 62.9 years [age range, 26-87 years]. Correlation tests were used and showed that preoperative information had no effect on statistical tests (data not shown).

\section{Procedures}

Twenty cerebral angiography procedures were live-recorded in our institution, between March and July 2010. All procedures were performed on an Allura Xper biplane X-ray system (Philips Medical Systems, Best, the Netherlands) with identical radiation exposure protocols. As detailed in Figure 1, four procedures were excluded: two for training purposes, one because a micro catheter was used, and a further one because additional vessels, such as carotid or vertebral arteries, were investigated. The mean number of investigated arteries was 
3.5. Sixteen procedures were therefore analyzed. In 8 cases, a senior neuroradiologist alone performed the procedure and in the remaining 8 cases the procedure was performed by a junior neuroradiologist with the supervision, and when necessary assistance, of a senior.

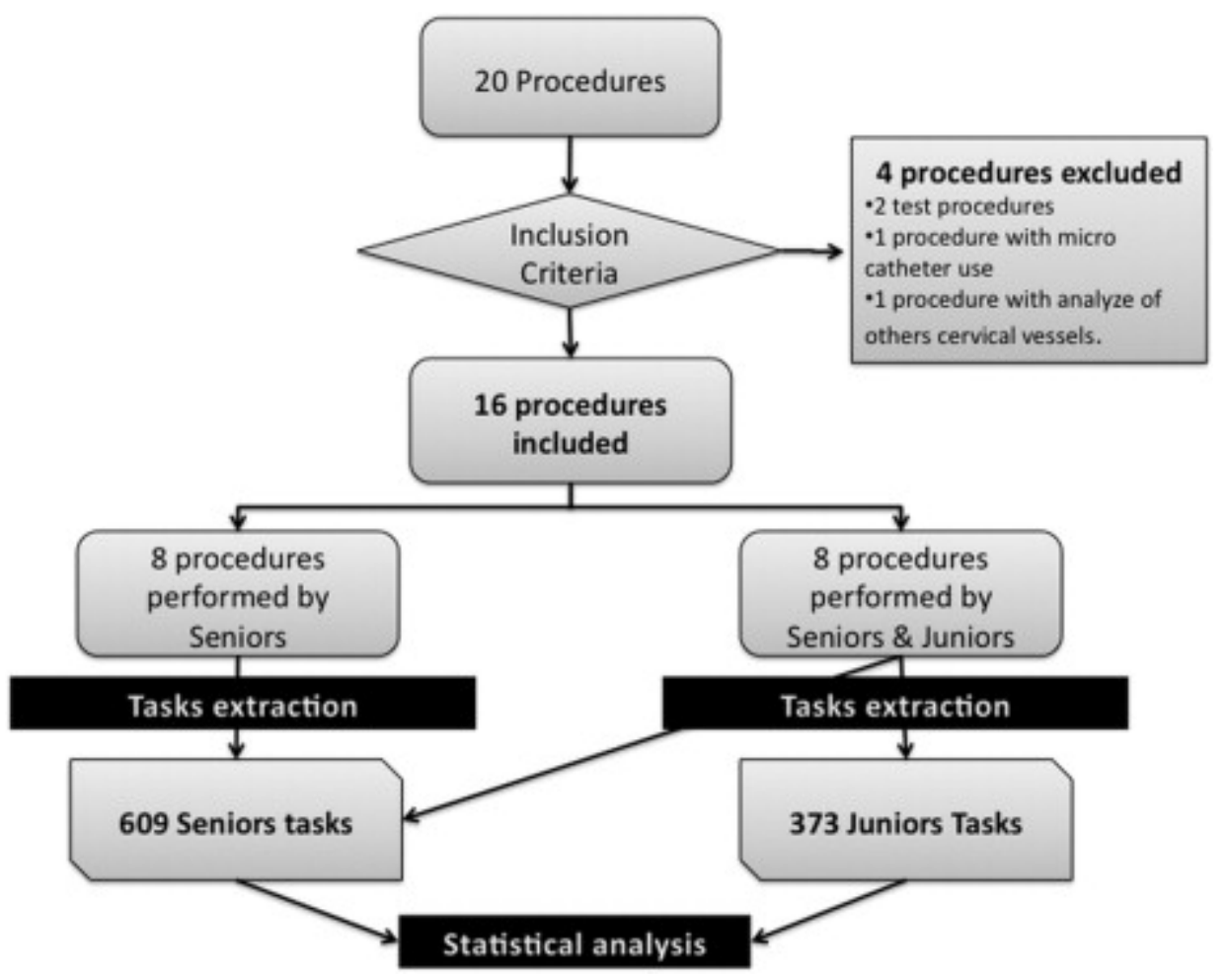

\section{Participants or Operators}

There were four participants or operators: two senior neuroradiologists, who had each performed over 500 cerebral angiographies, and two junior neuroradiologists. The juniors were residents at the end of their program and are now seniors in interventional neuroradiology. All the operators gave their approval for this study.

Methods

We adapted the methodology proposed and validated in the field of neurosurgery by Jannin et al. [9, 16] and Neumuth et al. [17, 18]. Four steps were necessary. First we defined the terminology used for describing the procedures. Second the procedures were described by an operator, resulting in a deconstructed surgical process models. Third the recorded data was further described in terms of phases. Fourth the data were analyzed. The terminology was defined and adapted from the standard terminology used to describe each task [19]. The terms 
were mainly chosen from the UMLS dataset and less frequently from the Mesh database. The data were acquired using a JAVA software application, the Surgical Workflow Editor [20]. The software was adapted to enable it to use the specific terminology for cerebral diagnostic angiographies. The descriptions of the procedures were recorded in the OR by an observer. The trained observer was a resident in neuroradiology. He had the possibility of recording the activities of one or two participants (when recording junior neuroradiologists) (Figure 2). During recording, the observer could also retrospectively request a senior neuroradiologist for help in identifying a task. In order to ensure correct task distribution the possibility was given to edit, verify, and correct the recording at the end of the procedure.
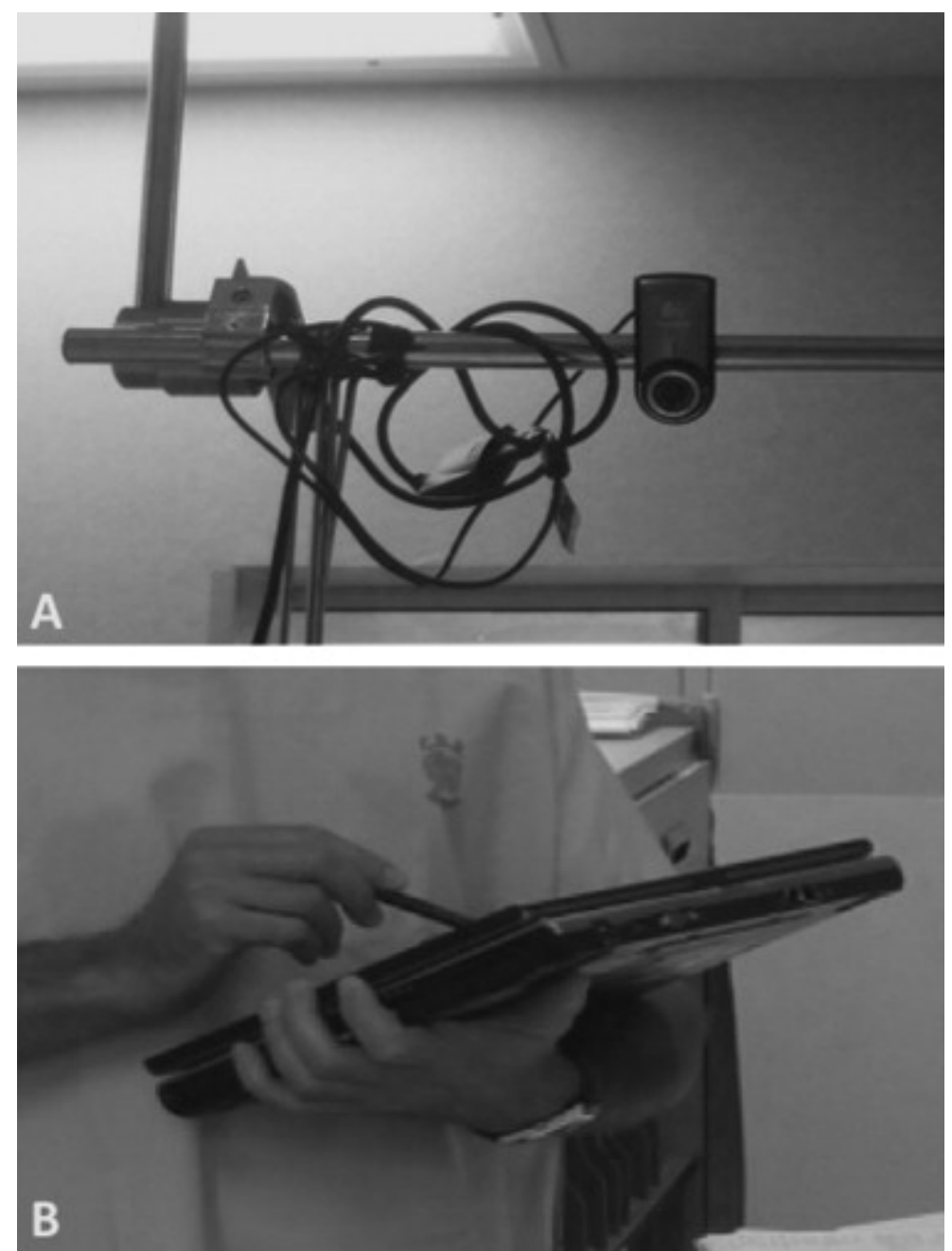

The video camera is focused on the operator's hands $(A)$. The observer codes the task thanks to the interface $(B)$. 
A typical cerebral angiography procedure was subdivided into three interventional phases: 1arterial access, 2-navigation and imaging, and 3-closure of the access. The navigation and imaging phase (phase 2) begins when the catheter is in the introducer and is complete when the catheter has been permanently removed. Recording a procedure with the dedicated software, consisted in selecting the appropriate phase of the procedure, and the surgical task. Each task was described by an action (i.e. navigate, insert, inject), an instrument (i.e. introducer, contrast agent, guide wire), and an anatomical structure (i.e. femoral artery, aorta, internal carotid artery) defining a triplet, for example insert/guide/internal carotid artery or navigate/catheter/ internal carotid Operators, start time, end time, and the duration of each task were recorded. The software created an XML file that included the list of recorded tasks (Figure 3).

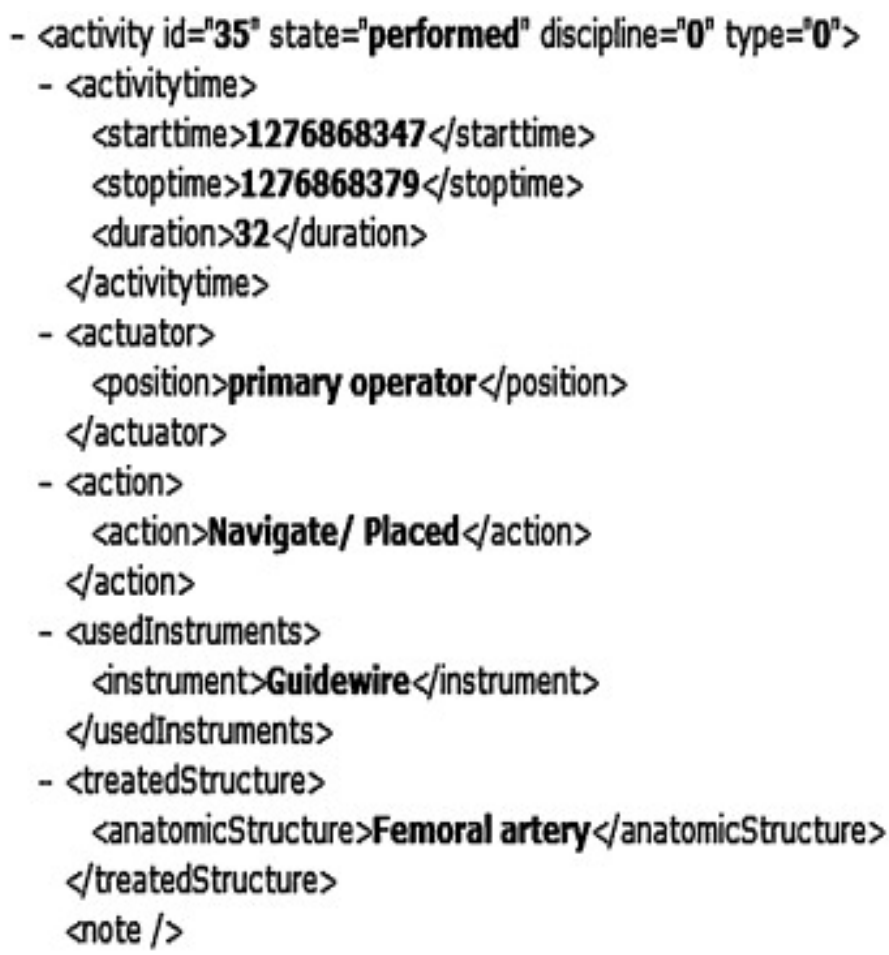

$X M L$ data: each triplet comprised an action, an instrument and an anatomical structure. In this case the primary operator performed task 35, which was identified as navigate/guidewire/femoral artery. The duration of the task was $32 \mathrm{~s}$. 
The duration was used as the metric for our analysis. Three parameters were isolated: the total duration of the entire cerebral angiography, the total duration of each task, and the number of times a task was repeated by the neuroradiologist. Additionally, we studied two other specific parameters that reflect the efficiency and competency of an interventional neuroradiologist: the use of fluoroscopy and movement of the X-ray system (table and tubes). For fluoroscopy use, we selected all triplets beginning with the action "navigate” (signifying fluoroscopy use) such as navigate/guide catheter/aorta or navigate/catheter/internal carotid artery. Total radiation dosage is the sum of radiation exposure during angiographic series and radiation exposure of fluoroscopy. In this study, we focused on radiation exposure for fluoroscopy to analyze the competencies of the operator. To study X-ray system movement, we selected the following triplet: move/table,tubes/ $X X X$ ( $X X X$ because it was not possible to choose an anatomical structure for this action). For this interaction with the table and the tubes, we also focused on the time spent before performing cerebral vessel imaging.

Analysis

The investigated parameters have been summarized in the Table I. After computing them, the Mann-Whitney U test was used for tasks time-based comparison between juniors and seniors. A P value of less than 0.05 was deemed significant.

Table 1.

Summary of the investigated parameters.

General parameter

Total duration of the entire procedure

\section{Non-specific tasks}

Mean time of tasks (including all recorded tasks in phase $2^{\underline{a}}$ ) 


\section{Specific tasks}

Total duration, number and mean duration of the following tasks

Fluoroscopy use

X-ray table and/or tube movements

X-ray table and/or tube movements before imaging

\section{a}

The cerebral angiography procedure was divided into three phases: 1: arterial access; 2: navigation and imaging; 3: closure of access.

\section{RESULTS}

Ninety-three different triplets were used to record the procedures. Junior neuroradiologists performed 373 tasks compared to 609 tasks for senior neuroradiologists. For both groups, the mean number of tasks per procedure was $75( \pm 24)$. A mean of 1.4 tasks per minute was recorded and the mean total duration of the procedure was $49( \pm 15)$ minutes.

Concerning the general parameters for the whole procedure, the total duration was statistically longer in case of participation of a junior $(\mathrm{P}=0.01)$ (Table II). For the non-specific tasks of the phase 2 , the duration per task was statistically longer for juniors $(\mathrm{P}=0.002)$, with a mean of 86 seconds versus 43 seconds for the seniors (Table II).

Table 2.

Total duration of tasks in the entire procedure, and mean duration of non-specific tasks (all tasks included) for the navigation and imaging phase (phase 2).

\section{Senior operators Junior operators $P$ value}

$$
\text { Mean } \pm \text { SD (s) Mean } \pm \text { SD (s) }
$$

$\begin{array}{lll}\text { Total duration of tasks (entire procedure) } 2032 \pm 387 & 2841 \pm 738 & 0.01\end{array}$

$\begin{array}{llll}\text { Mean duration per task (phase 2) } & 43 \pm 44 & 86 \pm 83 & 0.002\end{array}$


Concerning the first specific task, the total duration of tasks related to fluoroscopy use was statistically higher for the juniors ( $\mathrm{P}=0.002)$, with $173 \pm 131$ for the juniors and $454 \pm 192$ for the seniors (Table III). There was a statistical difference in the mean duration per task using fluoroscopy between the two groups $(\mathrm{P}=0.03), 37 \mathrm{~s}( \pm 12)$ for the juniors and $23 \mathrm{~s}( \pm 13)$ for the seniors. The number of times that the juniors used fluoroscopy was not statistically different from that of the seniors $(\mathrm{P}=0.11)$ : $12( \pm 4)$ in the junior group and $9( \pm 8)$ in the senior group.

Table 3.

Total duration, task duration and number of tasks using fluoroscopy.

\begin{tabular}{|c|c|c|c|}
\hline \multirow[t]{2}{*}{ Fluoroscopy use } & \multicolumn{3}{|c|}{ Senior operators Junior operators $P$ value } \\
\hline & Mean \pm SD & Mean \pm & \\
\hline Total duration (s) & $173 \pm 131$ & $454 \pm 192$ & 0.002 \\
\hline Duration of tas & $23 \pm 13$ & $37 \pm 12$ & 0.03 \\
\hline Number of tasks & $9 \pm 8$ & $12 \pm 4$ & 0.11 \\
\hline
\end{tabular}

For the second specific task, the total duration of tasks related to table or tube movement was higher in the junior group $(\mathrm{P}=0.02)$ (Table IV). There was no statistical difference for the duration per task involving table or tube movement between the two groups $(\mathrm{P}=0.08)$, 42s $( \pm 16)$ for the juniors and 30s $( \pm 10)$ for the seniors. The number of times that the juniors moved the table or tubes was not statistically different from that of the seniors $(\mathrm{P}=0.75)$.

Table 4.

Total duration, task duration and number of tasks involving X-ray table/tube movement.

\begin{tabular}{|c|c|c|c|}
\hline & Mean \pm SD & Mean \pm SD & \\
\hline Total duration (s) & $410 \pm 99$ & $660 \pm 210$ & 0.02 \\
\hline Duration of tasks (s) & $30 \pm 10$ & $42 \pm 16$ & 0.08 \\
\hline Number of tasks & $14 \pm 4$ & $17 \pm 5$ & 0.75 \\
\hline
\end{tabular}


For the third specific task, the total duration of tasks and the mean duration of tasks that involved moving the table and tubes before performing intracranial vessel imaging, was higher in the junior group and statistically different $(\mathrm{P}=0.02$ and $\mathrm{p}=0.03)$ (Table V).

Table 5.

Total duration, task duration and number of tasks involving table/tube movement before cerebral vessel imaging.

\section{Table/tube interactions before imaging cerebral vessels}

Total duration (s)

Duration of tasks (s)

Number of tasks

\begin{tabular}{lll}
$\begin{array}{c}\text { Senior } \\
\text { operators } \\
\text { Mean } \pm \text { SD }\end{array}$ & \multicolumn{1}{c}{$\begin{array}{c}\text { Junior } \\
\text { operators } \\
\text { Mean } \pm \text { SD }\end{array}$} & $\begin{array}{c}\boldsymbol{P} \\
\text { value }\end{array}$ \\
$299 \pm 104$ & $498 \pm 181$ & 0.02 \\
$37 \pm 13$ & $59 \pm 32$ & 0.03 \\
$9 \pm 3$ & $9 \pm 2$ & 0.71
\end{tabular}

\section{DISCUSSION}

Our approach allowed an objective time-based analysis and comparison of selected tasks used in cerebral diagnostic angiographies for two groups of operators [21]. The developed workflow task analysis tool allows quantitative study with high precision, as shown by the large amount of data recorded: 93 different triplets are used to record a cerebral angiography procedure; a mean of $75( \pm 24)$ tasks during the entire procedure and a mean of 1.4 recorded tasks per minute. For comparison, in a workflow task analysis study about nerve and facet blocks in interventional radiology, on average, 22 tasks were recorded [12] compared to the mean of $75( \pm 24)$ tasks per procedure in our study. Moreover, for each task, our software computed the total duration of each task and the number of times a task was performed.

The result shows that a junior takes basically twice as long as a senior to perform a task: the duration per task is statistically higher for the junior group: on average 43 seconds for the seniors versus 86 seconds for the juniors. Concerning the more specific tasks probably more related to operator skills, the use of fluoroscopy and the interactions with the table and/or the 
tubes are interesting. The total and mean duration of tasks that use fluoroscopy were longer in the junior as well as the tasks for table/tube movement before vessel imaging. Even if the cerebral angiography is a well-standardized procedure, we suppose that those results are the reflect of operator experience leading to decrease the need of fluoroscopy and table movement to perform the angiography.

The choice of the duration of tasks as the principal metric has advantages and limitations. One advantage is that the record includes all the tasks. As the precision of the recording is high, all tasks are studied even if they are short. It is therefore possible to show the impact of a minor variable on the duration or repetition of tasks. Another advantage is the opportunity of recording various operators simultaneously. This is particularly useful in a study comparing junior versus senior practitioners, because the junior's tasks must be assessed despite the senior's influence/participation. This is the reason why; to our point of view the best criterion to use to compare the durations is the mean duration per task because the concerned task is operator specific. However, it is very difficult and almost impossible to completely suppress the influence of the senior and especially the vocal influence. One limiting factor of our approach is that we analyzed only the tasks' duration. If this technique is distinguished from a simple temporal analysis, especially since it allows us to focus on "active tasks", it could be said that time is not the best criterion for assessing competence and that time-action-analysis does not provide any measure of quality. We are currently working on using other metrics for analysis, measuring for instance the standardization of the procedures. The last limitation is the manual acquisition and recording of the interventional processes responsible of the small number of the recorded procedures. This is, in fact, a time-consuming technique that requires the presence of a trained observer in the radiology OR. Some recent development in surgery make us thinking that the recording will one day be performed without human intervention but will be based on automatically detection of operator tasks by using captors and/or 
detectors [22, 23]. In the future, this method of workflow task analysis could be used to highlight a task that the operator never performs, or is presumed to be difficult or executed incorrectly or more slowly.

One-way to assess operators in training will be to repeat the recordings during residency or fellow program for example and use our measurements to analyse the learning curve. Another possible application of this approach could be to assess the effects of training sessions on simulators in diagnostic or therapeutic procedures. After highlighting such tasks, it would be possible to train the operator in faster and safer practices. However, even if our attention has been focused mainly on the operator, the operator is not necessarily the study's only object in a workflow task analysis. By using the same approach, the influence of patient vessels' anatomy, therapeutic techniques, and radiation exposure protocols could be studied. Using this method, further work is underway to study the differences of practice such as standardization / repeatability of gesture in a population of procedures [24].

\section{CONCLUSIONS}

We showed the feasibility of a new approach based on workflow time-based task analysis that allows comparison of junior and senior tasks' durations. It could serve as a future evaluation tool to improve quality and safety of procedures by highlighting the tasks on which the junior should focus (i.e. table/tubes manipulation, patients head positioning before imaging).

Conflict of interest: None

\section{FIGURES}

Figure 1. Flow Chart

Figure 2. A video camera focused on operator's hands (A). The observer codes the task thanks to the interface (B). 
Figure 3. XML data: Each triplet is composed by the association of an action, a tool and an anatomical structure. For example, the primary operator performed the task 35 . This task wasNavigate/Guidewire/Femoral Artery. The duration of this task was 32 seconds. 


\section{REFERENCES}

1. Ahmed K, Keeling A, Khan R, Ashrafian H, Arora S, Nagpal Ket al. What Does Competence Entail in Interventional Radiology? CardioVascular and Interventional Radiology 2010;33:3-10

2. Aggarwal R, Darzi A. Technical-Skills Training in the 21st Century. N Engl J Med 2006;355:2695-96

3. Duncan JR. Strategies for improving safety and quality in interventional radiology. J Vasc Interv Radiol 2008;19:3-7

4. Krauss A, Muensterer OJ, Neumuth T, Wachowiak R, Donaubauer B, Korb Wet al. Workflow analysis of laparoscopic Nissen fundoplication in infant pigs- a model for surgical feedback and training. J Laparoendosc Adv Surg Tech A 2009;19 Suppl $1: S 117-22$

5. Lemke HU, Trantakis C, Köchy K, Müller A, Strauss G, Meixensberger J. Workflow analysis for mechatronic and imaging assistance in head surgery. International Congress Series 2004;1268:830-35

6. Bakker NH, Tanase D, Reekers JA, Grimbergen CA. Evaluation of vascular and interventional procedures with time-action analysis: a pilot study. J Vasc Interv Radiol 2002;13:483-8

7. Den Boer KT, Straatsburg IH, Schellinger AV, de Wit LT, Dankelman J, Gouma DJ. Quantitative analysis of the functionality and efficiency of three surgical dissection techniques: a time-motion analysis. J Laparoendosc Adv Surg Tech A 1999;9:389-95

8. Mehta NY, Haluck RS, Frecker MI, Snyder AJ. Sequence and task analysis of instrument use in common laparoscopic procedures. Surgical Endoscopy 2002;16:28085 
9. Jannin P, Raimbault M, Morandi X, Riffaud L, Gibaud B. Model of surgical procedures for multimodal image-guided neurosurgery. Comput Aided Surg 2003;8:98-106

10. Lindisch D, Neumuth T, Burgert O, Spies J, Cleary K. Workflow in interventional radiology: uterine fibroid embolization (UFE). In: Medical Imaging 2008: PACS and Imaging Informatics. San Diego, CA, USA: SPIE, 2008:69190Y-10

11. Riffaud L, Neumuth T, Morandi X, Trantakis C, Meixensberger J, Burgert Oet al. Recording of surgical processes: a study comparing senior and junior neurosurgeons during lumbar disc herniation surgery. Neurosurgery 2010;67:325-32

12. Siddoway D, Ingeholm ML, Burgert O, Neumuth T, Watson V, Cleary K. Workflow in interventional radiology: nerve blocks and facet blocks. In: Medical Imaging 2006: PACS and Imaging Informatics. San Diego, CA, USA: SPIE, 2006:61450B-8

13. Neumuth T, Jannin P, Schlomberg J, Meixensberger J, Wiedemann P, Burgert O. Analysis of surgical intervention populations using generic surgical process models. Int J Comput Assist Radiol Surg 2010;

14. Neumuth $T$, Jannin P, Strauss G, Meixensberger J, Burgert O. Validation of knowledge acquisition for surgical process models. J Am Med Inform Assoc 2009;16:72-80

15. Neumuth T, Trantakis C, Riffaud L, Strauss G, Meixensberger J, Burgert O. Assessment of technical needs for surgical equipment by surgical process models. Minim Invasive Ther Allied Technol 2009;18:341-9

16. Jannin P, Morandi X. Surgical models for computer-assisted neurosurgery. Neuroimage 2007;37:783-91 
17. Neumuth T, Strauß G, Meixensberger J, Lemke H, Burgert O. Acquisition of Process Descriptions from Surgical Interventions. In: Database and Expert Systems Applications, 2006:602-11

18. Neumuth T, Durstewitz N, Fischer M, Strauss G, Dietz A, Meixensberger Jet al. Structured recording of intraoperative surgical workflows. In: Medical Imaging 2006: PACS and Imaging Informatics. San Diego, CA, USA: SPIE, 2006:61450A-12

19. (UMLS $\left.{ }^{\circledR}\right)$ UMLS. Unified Medical Language System ${ }^{\circledR}\left(\mathrm{UMLS}^{\circledR}\right)$. In:

20. Neumuth, R M, Jannin P, J M, O B, T Net al. The tool landscap for surgical workflow analysis. In: Computer Assisted Medical and Surgetical Interventions, 2007:199-204

21. Darzi A, Smith S, Taffinder N. Assessing operative skill. Needs to become more objective. BMJ 1999;318:887-8

22. Bouget D, Lalys F, Jannin P. Surgical tools recognition and pupil segmentation for cataract surgical process modeling. Stud Health Technol Inform 2012;173:78-84

23. Lalys F, Riffaud L, Bouget D, Jannin P. An application-dependent framework for the recognition of high-level surgical tasks in the OR. Medical image computing and computer-assisted intervention : MICCAI International Conference on Medical Image Computing and Computer-Assisted Intervention 2011;14:331-8

24. Forestier G, Lalys F, Riffaud L, Trelhu B, Jannin P. Classification of surgical processes using dynamic time warping. Journal of biomedical informatics 2012;45:255-64 